\title{
How Flagella Expression May be Regulated by the Carbon and Energy Source?
}

\author{
Anibal R. Lodeiro* \\ Laboratory of Interactions between Rhizobia and Soybean (LIRyS). IBBM-Faculty of Sciences. National University of \\ La Plata and CONICET CCT-La Plata, Argentina
}

Received: August 29, 2014; Accepted: September 26, 2014; Published: October 14, 2014

*Corresponding author: Anibal R. Lodeiro, IBBM-Faculty of Exact Sciences, UNLP-CONICET. Calles 47 y 115 (1900) La Plata, Argentina, Tel: +54-221422-9777; E-mail: lodeiro@biol.unlp.edu.ar

\begin{abstract}
Bradyrhizobium diazoefficiens has two flagellar systems: a subpolar, constitutive system and a lateral, inducible system. Contrary to other bacterial species, the lateral system is induced in liquid medium in response to the carbon and energy source. Since both flagella are moved by the proton-motive force, a relationship between the energy status of the cell and the signal that triggers lateral flagella expression might exist. Here I discuss how this relationship may control the induction of the lateral flagellar system, and its implicancies for improvement of Bradyrhizobium-based inoculants for soybean plants.
\end{abstract}

Keywords: Flagella; Bradyrhizobium diazoefficiens; Soyabean; Symbiosis

\section{Introduction}

Bradyrhizobium is a soil bacterial genus that includes several species of importance due to their use as biofertilizers for soybean crops worldwide. Among these species, B. diazoefficiens and $B$. japonicum stand out as being the most widely employed.

These bacteria fix atmospheric $\mathrm{N}_{2}$ in symbiosis with soybean plants, by reducing $\mathrm{N}_{2}$ to $\mathrm{NH}_{4}{ }^{+}$in a reaction catalyzed by bradyrhizobial nitrogenase. The $\mathrm{NH}_{4}^{+}$thus produced is supplied as $\mathrm{N}$-source to the plant, in such a rate that all its $\mathrm{N}$-needs may be satisfied. To this end, the Bradyrhizobium bacteria are inoculated to soybean seeds before sowing with the aim that these bacteria infect the roots and develop the $\mathrm{N}_{2}$-fixing symbiosis inside them. Since the symbiosis only occurs in specialized organs in the roots, seed-inoculated bacteria need moving from the site of inoculation to the sites of infection, and this movement has to occur in the soil, a porous and tortuous medium, which not always contain water enough for bacterial swimming. Therefore, the study of bradyrhizobial motility is of prime importance to improve this ecologically sustainable technology for soybean fertilization.

B. diazoefficiens USDA 110 is the type strain of this species [1], and its genome was completely sequenced in 2002 [2]. In addition, this strain is the most studied biochemically, genetically, and physiologically, as well as in the relevant aspects of its symbiosis with soybean plants. The motility of this strain was characterized in two kinds of bacterial movement: swimming [3-
5] and swarming [6]. Both movements are propelled by flagella, while other movements such as e.g. twitching or gliding were not reported. Although there exists evidence of the presence of pili, which constitute the device required for twitching, these appendages were studied only for their role in cell adhesion [7].

Remarkably, B. diazoefficiens USDA 110 possesses two entirely different flagellar systems: a subpolar system and a lateral system [4-6]. These systems are encoded in different gene clusters, and it seems that each one possesses its own regulatory system for the control if its expression. Indeed, the expression of the subpolar system seems constitutive in planktonic cells, while the lateral system is inducible [5,6]. Induction of the lateral system was observed as obeying to the carbon and energy source of the growth medium: when the sole carbon and energy source is arabinose, the lateral system is expressed, but it is inhibited when the only carbon and energy source is mannitol [6]. Although several other bacterial species are known to possess inducible lateral flagellar systems, in general the inducer is the medium viscosity or the proximity of a surface, which are perceived by the polar/subpolar flagellar system that under these circumstances behaves as a mechanosensor [8]. However, B. diazoefficiens is the only example known where the lateral flagellar system is induced by the carbon and energy source, and therefore the identity of the signal transducer is a complete enigma.

\section{Structure and Rotation of Flagella}

The flagellum consists in three main structures: the flagellar filament, the hook, and the basal body, which contains the motor [9]. Although the flagellar motor was not studied in $B$. diazoefficiens, there exists a great deal of knowledge in other species, in particular Escherichia coli [10]. The flagellar motor is embedded in the inner cell membrane and has two main rings: a stator formed mainly by the proteins MotA and MotB, and a rotor to which the rest of the flagellum is attached. The rotor is composed mainly by the proteins FliF, FliG, FliM and FliN, which play a central role in flagellar rotation. The Protonmotive Force (PMF) that is generated during cell respiration is the energy source for flagellar motor rotation. Protons pass from the periplasmic space towards the cytoplasm through a channel formed between FliG and MotA/MotB. Recent studies indicate that the rotor contains a ring of 26 FliG subunits faced against an external ring of MotA/ 
MotB subunits in such a way that an array of negatively charged amino acids in MotA, MotB and FliG interact with the protons that traverse the channel, producing changes in the conformations of the ring proteins, allowing movement of the rotor. These amino acids are disposed in such a way that the passage of protons from the periplasm to the cytoplasm moves the rotor against the stator thus producing a torque sufficient to impulse the cell body into the liquid environment [10-12].

In general, bacterial species that possess two flagellar systems use the PMF to move one of the flagella, while the other is moved by a $\mathrm{Na}^{+}$gradient that is also formed between the periplasm and the cytoplasm. However, in B. diazoefficiens both flagellar systems seem to be moved by the PMF [4], thus sharing this energy source with the synthesis of ATP. Ultimately, the PMF comes from the oxidation of organic carbon and energy sources, and therefore the availability of energy in the cell might be in connection with the regulation of the lateral flagellum expression by the carbon and energy source.

\section{Catabolism of Arabinose and Mannitol}

L-arabinose is catabolized through a pathway that resembles the Entner-Doudoroff (ED) pathway for catabolism of hexoses $[13,14]$ (Figure 1). The first step is oxidation of L-arabinose to
L-arabonate with formation of one mole of NADH per mole of L-arabinose. Then, L-2-keto 3-deoxy arabonate is formed, which splits in pyruvate and glycolaldehyde in a reaction catalyzed by an aldolase. The pyruvate continues through TCA cycle, while glycolaldehyde is oxidized in two sequential steps of NADHproducing reactions to glyoxylate, which is finally converted to formate and oxidized to $\mathrm{CO}_{2}$ with production of another $\mathrm{NADH}$ [13-15]. Hence, a total of eight NADH moles plus one $\mathrm{FADH}_{2}$ mole are produced per mole of arabinose completely oxidized to $\mathrm{CO}_{2}$.

D-mannitol is oxidized to fructose with production of one NADH mole per mole of mannitol in a reaction catalyzed by mannitol dehydrogenase [16]. Then, the fructose produced may be catabolized by the ED or the Emden-Meyerhof-Parnas (EMP) pathways [17], or the Pentose-Phosphate (PP) pathway [18] with production of 10 additional NADH moles plus two $\mathrm{FADH}_{2}$ moles (ED, EMP pathways), or 11 additional NADH moles plus two $\mathrm{FADH}_{2}$ moles (PP pathway) in the complete oxidation of one mole of fructose to $\mathrm{CO}_{2}$. In Figure 1, the yields of NADH plus $\mathrm{FADH}_{2}$ with arabinose are compared with those with mannitol catabolized by the ED pathway as an example.

Thus, assuming that $10 \mathrm{H}^{+}$moles are passed to the periplasm per mole of NADH oxidized and $6 \mathrm{H}^{+}$moles are passed per mole of

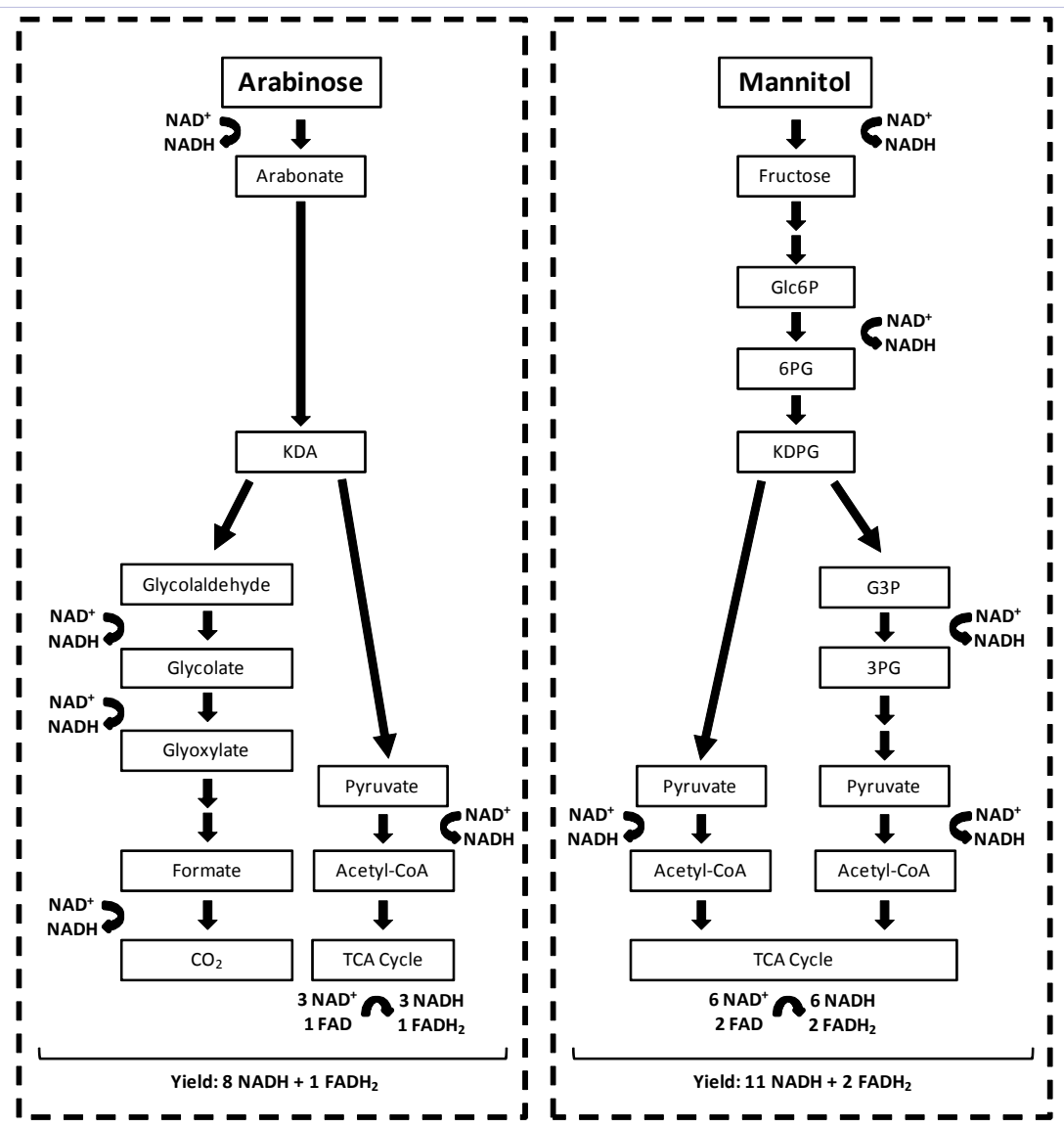

Figure 1: Comparison of the catabolism of arabinose (left) and mannitol (through the ED pathway, right) in Bradyrhizobium diazoefficiens with emphasis in the reactions where reducing power is generated. KDA: 2-keto-3-deoxyarabonate; Glc6P: glucose-6-phosphate; 6PG: 6- phosphogluconate; KDPG: 2-keto-3-deoxyphosphogluconate; G3P: glyceraldehyde-3-phosphate; 3PG: 3-phosphoglycerate. 
$\mathrm{FADH}_{2}$ oxidized, it results that $86 \mathrm{H}^{+}$moles are passed per mole of arabinose or 122-132 $\mathrm{H}^{+}$moles are passed per mole of mannitol completely oxidized to $\mathrm{CO}_{2}$. Despite this higher yield of mannitol with respect to arabinose, $\mathrm{O}_{2}$ consumption rate was reported many years ago as roughly twice higher with arabinose than with mannitol [19], but these observations should be repeated with B. diazoefficiens USDA 110, and modern culture conditions and analytic technologies. Anyway, these data indicate that although the molar yield of $\mathrm{H}^{+}$is lower with arabinose than with mannitol, the net rate of $\mathrm{H}^{+}$passage from cytoplasm to periplasm per mole of carbon source oxidized still might be around 50\% higher with arabinose. Since growth rates in minimal medium with arabinose or mannitol are similar [6], energy consumption rates for growth should also be similar, and according to the above estimates, a higher PMF may remain available for maintenance functions when arabinose is the carbon and energy source.

\section{Perspectives}

We could envisage that the cell senses the conditions in which PMF is sufficient for ATP synthesis and motion of both flagella systems at the same time and only if these conditions are met, lateral flagella expression is allowed. The conditions need not necessarily involve high viscosity of the medium because the induction of the lateral flagellar system by arabinose was observed in liquid medium. If arabinose is present in the root exudates near the infection sites [3], the expression of lateral flagella in response to this carbohydrate might be useful for the bacteria to stabilize their swimming direction towards such sites [20]. To respond to the cell energy status, the regulator(s) of lateral flagella expression should perform some measure of the PMF. There exist some ways of measuring PMF in connection with motility. For instance, a group of chemoreceptors specialized in sensing the energy status of the cell is known. These chemoreceptors bind FAD and are able to sense the redox state of the electron transport chain to elicit energy taxis, i.e. the orientation of the bacterial cell swimming towards an energy-rich environment [21]. Another candidate is the Phosphotransferase System (PTS), which also participates in chemotaxis [22]. Despite these systems being known as sensors of energy status in relation with motility, they do not display a clear relationship with the control of transcription or translation. Whether these systems, or a yet unknown signal transduction system, play a role in the control of lateral flagellar expression in response to the carbon and energy source is a research issue that might provide new knowledge about regulation of energy use in bacteria.

This issue is of special importance in the Bradyrhizobiumsoybean symbiosis. For instance, in Argentina more than 20 million hectares are cultivated with soybean, and $94 \%$ of producers use Bradyrhizobium-based inoculants to achieve $\mathrm{N}$-nutrition through biological $\mathrm{N}_{2}$ fixation in their crops [23]. Motility of Bradyrhizobium bacteria in the soil is essential to achieve a successful symbiotic interaction [5] and therefore, understanding the control of motility and its stimulation by rootexuded compounds is one key for the development of improved inoculants for agriculture.

\section{Acknowledgements}

The author is member of the Scientific Career of CONICET, Argentina, and his work is financed by ANPCyT, CONICET, and UNLP, all from Argentina.

\section{References}

1. Delamuta JR, Ribeiro RA, Ormeño-Orrillo E, Melo IS, Martínez-Romero $\mathrm{E}$ and Hungria M. Polyphasic evidence supporting the reclassification of Bradyrhizobium japonicum group Ia strains as Bradyrhizobium diazoefficiens sp. nov. Int J Syst Evol Microbiol. 2013; 63: 3342-51. doi: 10.1099/ijs.0.049130-0.

2. Kaneko T, Nakamura Y, Sato S, Minamisawa K, Uchiumi T, Sasamoto $\mathrm{S}$, et al. Complete genomic sequence of nitrogen-fixing symbiotic bacterium Bradyrhizobium japonicum USDA110. DNA Res. 2002; 9(6): 189-97.

3. Barbour WM, Hattermann DR and Stacey G. Chemotaxis of Bradyrhizobium japonicum to soybean exudates. Appl Environ Microbiol. 1991; 57(9): 2635-39.

4. Kanbe M, Yagasaki J, Zehner S, Göttfert M and Aizawa S. Characterization of two sets of subpolar flagella in Bradyrhizobium japonicum. J Bacteriol. 2007; 189(3): 1083-89.

5. Althabegoiti MJ, Covelli JM, Perez-Gimenez J, Quelas JI, Mongiardini EJ, Lopez MF, et al. Analysis of the role of the two flagella of Bradyrhizobium japonicum in competition for nodulation of soybean. FEMS Microbiol Lett. 2011; 319(2): 133-39. doi: 10.1111/j.1574-6968.2011.02280.x.

6. Covelli JM, Althabegoiti MJ, Lopez MF and Lodeiro AR. Swarming motility in Bradyrhizobium japonicum. Res Microbiol. 2013; 164(2): 136-44. doi: 10.1016/j.resmic.2012.10.014.

7. Vesper SJ and Bauer WD. Role of pili (Fimbriae) in attachment of bradyrhizobium japonicum to soybean roots. Appl Environ Microbiol. 1986; 52(1): 134-41.

8. Merino S and Tomás JM. Lateral Flagella Systems. In: Jarrell KF, editor. Pili and Flagella. Current Research and Future Trends. Kingston, UK: Caister Academic Press; 2009. p 173-90.

9. McCarter LL. Regulation of flagella. Curr Opin Microbiol. 2006; 9(2): 180-86.

10. Berg HC. The rotary motor of bacterial flagella. Annu Rev Biochem. 2003; 72: 19-54.

11. Stock D, Namba K and Lee LK. Nanorotors and self-assembling macromolecular machines: the torque ring of the bacterial flagellar motor. Current Opin Biotechnol. 2012; 23(4): 545-54. doi: 10.1016/j. copbio.2012.01.008.

12. Attmannspacher U, Scharf B and Schmitt R. Control of speed modulation (chemokinesis) in the unidirectional rotary motor of Sinorhizobium meliloti. Mol Microbiol. 2005; 56(3): 708-18.

13. Pedrosa FO and Zancan GT. L-Arabinose metabolism in Rhizobium japonicum. J Bacteriol. 1974; 119(1): 336-38.

14. Watanabe S, Shimada N, Tajima K, Kodaki T and Makino K Identification and characterization of L-arabonate dehydratase, L-2-keto-3-deoxyarabonate dehydratase, and L-arabinolactonase involved in an alternative pathway of L-arabinose metabolism. Novel evolutionary insight into sugar metabolism. J Biol Chem. 2006; 281(44): 33521-36

15. Koch M, Delmotte N, Ahrens CH, Omasits U, Schneider K, Danza F, et al. A link between arabinose utilization and oxalotrophy in Bradyrhizobium japonicum. Appl Environ Microbiol. 2014; 80(7): 2094-101. 
16. Kuykendall LD and Elkan GH. Some features of mannitol metabolism in Rhizobium japonicum. J Gen Microbiol. 1977; 98(1): 291-95.

17. Mulongoy K and Elkan GH. Glucose catabolism in two derivatives of a Rhizobium japonicum strain differing in nitrogen-fixing efficiency. J Bacteriol. 1977; 131(1): 179-87.

18. Sosa-Saavedra F, León-Barrios M and Pérez-Galdona R. Pentose phosphate pathway as the main route for hexose catabolism in Bradyrhizobium sp. lacking Entner-Doudoroff pathway. A role for $\mathrm{NAD}^{+}$-dependent 6-phosphogluconate dehydrogenase (decarboxylating). Soil Biol Biochem. 2001; 33(3): 339-43. doi: 10.1016/S0038-0717(00)00146-2

19. Thorne DW and Burris RH. Respiratory enzyme systems in symbiotic nitrogen fixation: III. The respiration of Rhizobium from legume nodules and laboratory cultures. J Bacteriol. 1940; 39(2): 187-96.

20. Bubendorfer S, Koltai M, Rossmann F, Sourjik V and Thormann KM.
Secondary bacterial flagellar system improves bacterial spreading by increasing the directional persistence of swimming. Proc Natl Acad Sci USA. 2014; 111(31):11485-90. doi: 10.1073/pnas.1405820111.

21. Alexandre G. Coupling metabolism and chemotaxis-dependent behaviours by energy taxis receptors. Microbiology. 2010; 156: 228393. doi: 10.1099/mic.0.039214-0.

22. Neumann S, Grosse K and Sourjik V. Chemotactic signaling via carbohydrate phosphotransferase systems in Escherichia coli. Proc Natl Acad Sci USA. 2012; 109(30): 12159-64. doi: 10.1073/ pnas.1205307109.

23. Piccinetti C, Arias N, Ventimiglia L et al. Efectos Positivos de la Inoculación de Soja sobre la Nodulación, la FBN y en los Parámetros de Producción del Cultivo. In Albanesi AS (ed). Microbiología Agrícola. Un aporte de la Investigación en Argentina, 2da ed. Tucumán, Argentina. Magna Publicaciones, 2013;283-97. 